\title{
Geodesics avoiding open subsets in surfaces of negative curvature
}

\author{
Buyalo, S ; Schroeder, Viktor ; Walz, M
}

\begin{abstract}
We prove existence and non-existence results for geodesics avoiding $a$-separated sets on a surface of negative curvature.
\end{abstract}

DOI: https://doi.org/10.1017/S0143385700000559

Posted at the Zurich Open Repository and Archive, University of Zurich ZORA URL: https://doi.org/10.5167/uzh-22075

Journal Article

Originally published at:

Buyalo, S; Schroeder, Viktor; Walz, M (2000). Geodesics avoiding open subsets in surfaces of negative curvature. Ergodic Theory and Dynamical Systems, 20(4):991-1006.

DOI: https://doi.org/10.1017/S0143385700000559 


\title{
Geodesics avoiding open subsets in surfaces of negative curvature
}

\author{
SERGEI BUYALO $\dagger$, VIKTOR SCHROEDER $\ddagger$ and MICHEL WALZ $\ddagger$ \\ $\dagger$ St. Petersburg Branch, V. A. Steklov Institute of Mathematics (POMI), \\ Russian Academy of Sciences, Fontanka 27, St. Petersburg, 191011, Russia \\ (e-mail: buyalo@pdmi.ras.ru) \\ \$ Mathematisches Institut, Universität Zürich, Winterthurer Str. 190, CH-8057 Zürich, \\ Switzerland \\ (e-mail: \{vschroed,mwalz\}@math.unizh.ch)
}

(Received 11 June 1998 and accepted in revised form 9 June 1999)

\begin{abstract}
We prove existence and non-existence results for geodesics avoiding $a$-separated sets on a surface of negative curvature.

\section{Introduction}

This paper is motivated by the following question:

Suppose that $M$ is a compact surface equipped with a Riemannian metric of pinched negative curvature $-b^{2} \leq K \leq-a^{2}<0$. Does there exist a proper closed subset $W$ of the unit tangent bundle $S M$ invariant under the geodesic flow $\varphi^{t}: S M \rightarrow S M$ such that $\pi(W)=M$, where $\pi: S M \rightarrow M$ is the footpoint projection?
\end{abstract}

Note that by ergodicity $W$ has Liouville measure zero in $S M$. In the special case of constant curvature, Burns and Pollicott [BP] gave a positive answer. The existence of $W$ follows from Theorem 1.1.

THEOREM 1.1. (Burns, Pollicott) Let $M=\Gamma \backslash \mathbb{H}^{2}$ be a quotient of the hyperbolic plane $\mathbb{H}^{2}$ by a discrete torsion-free group $\Gamma$ of isometries, and let $v_{0} \in S M$ be a non-recurrent vector. Then there exists an $\varepsilon>0$ and for every point $x \in M$ a unit speed geodesic $\gamma_{x}: \mathbb{R} \rightarrow M$ with $\gamma_{x}(0)=x$ such that $d^{1}\left(\dot{\gamma}(t), v_{0}\right) \geq \varepsilon$ for all $t \in \mathbb{R}$, where $d^{1}$ denotes the standard (Sasaki) metric on $S M$.

As a consequence, the set $W:=\left\{v \in S M \mid d^{1}\left(\varphi^{t}(v), v_{0}\right) \geq \varepsilon \forall t \in \mathbb{R}\right\}$ is a proper closed $\varphi^{t}$-invariant set with $\pi(W)=M$. Actually, a modification of the argument of Burns and Pollicott (see $\S 2$ ) shows that the non-recurrence condition on $v_{0}$ is not necessary. The theorem is an easy consequence of the existence of geodesics avoiding certain open subsets in hyperbolic space, which can be stated as in Theorem 1.2 below. Before we formulate our results it is convenient to introduce the following notation. 
Definition. A subset $\Omega$ of a metric space $(X, d)$ is called $a$-separated (for some $a \in$ $(0, \infty))$ if $d\left(x, x^{\prime}\right) \geq a$ for all distinct $x, x^{\prime} \in \Omega$.

THEOREM 1.2. Let $\mathbb{H}^{2}$ be the hyperbolic 2-plane with a distinguished point o $\in \mathbb{H}^{2}$. Let $\Omega \subset X$ be an a-separated subset with $d(o, x) \geq$ a for all $x \in \Omega$. Then there exists $\varepsilon=\varepsilon(a)>0$ and a geodesic $\gamma: \mathbb{R} \rightarrow \mathbb{H}^{2}$ with $d(\gamma(\mathbb{R}), \Omega) \geq \varepsilon$ and $\gamma(0)=o$.

This result was proved by Burns and Pollicott under the additional assumption $a \geq a_{0}$, where $a_{0}$ is a certain universal lower bound. We give a different argument to avoid this assumption. We mention that Theorem 1.2 and the Burns-Pollicott result have a generalization to rank-1 symmetric spaces.

The first main result of this paper is the construction of an example that shows that Theorem 1.2 depends essentially on the fact that $\mathbb{H}^{2}$ is a symmetric space and that a corresponding result fails in the variable curvature case.

THEOREM 1.3. Given $\delta>0$ and $a>0$, the space $\mathbb{R}^{2}$ can be equipped with a complete smooth Riemannian metric $g$ of sectional curvature $-1-\delta \leq K \leq-1+\delta$ such that there exists an a-separated subset $\Omega \subset \mathbb{R}^{2}$ enjoying the properties:

(1) $\quad d(o, x) \geq$ a for all $x \in \Omega$;

(2) if $\gamma: \mathbb{R} \rightarrow\left(\mathbb{R}^{2}, g\right)$ is a complete geodesic with $\gamma(0)=o$, then $d(\gamma(\mathbb{R}), \Omega)=0$.

We remark that our example does not cover any compact or finite volume surface. However, it shows that the methods of Burns and Pollicott cannot be generalized to the variable curvature case.

We note that in Theorems 1.2 and 1.3 it is essential to consider complete geodesics $\gamma: \mathbb{R} \rightarrow X$ with prescribed origin $o=\gamma(0)$.

A closely related problem is the study of geodesic rays $\gamma:[0, \infty) \rightarrow X$ starting at a prescribed point $o$. In this context one obtains two different analogs of Theorem 1.2. In the first generalization we need a curvature pinching, in the second we need only an upper curvature bound, but a lower bound on the separation number $a$.

THEOREM 1.4. Let $b \geq 1$ and $a>0$ be given. Then there exists a positive constant $\varepsilon=\varepsilon(b, a)$ with the following property.

Let $X$ be a complete simply connected surface with curvature $-b^{2} \leq K \leq-1$ and let $\Omega \subset X$ be an a-separated subset. Then for each $o \in X, d(o, \Omega) \geq a / 2$, there exists $a$ geodesic ray $\gamma:[0, \infty) \rightarrow X$ with $d(\gamma([0, \infty)), \Omega) \geq \varepsilon$ and $\gamma(0)=o$.

The second generalization is based on arguments of [BP].

THEOREM 1.5. For every $a>\ln 2$ there exists an $\varepsilon>0$ with the following property.

Let $X$ be a complete simply connected surface with curvature $K \leq-1$, and let $\Omega \subset X$ be an a-separated subset. Then for each $o \in X, d(o, \Omega) \geq a / 2$ there exists a geodesic ray $\gamma:[0, \infty) \rightarrow X$ with $d(\gamma([0, \infty)), \Omega) \geq \varepsilon$ and $\gamma(0)=o$.

In this theorem, we do not suppose that $X$ is smooth, and the condition $K \leq-1$ is understood in the usual Alexandrov comparison sense. Moreover, Theorem 1.5 is true for an arbitrary Hadamard space $X$ with curvature $K \leq-1$ and extendable geodesics whose space of directions at every point $x \in X$ contains at least three elements (see $\S 4$ ). Thus 
Theorem 1.5 is true for $\mathbb{R}$-trees, but the last condition excludes simplicial trees which give easy counterexamples.

Surprisingly, the lower bound $\ln 2=0.693 \ldots$ in Theorem 1.5 cannot be essentially improved. Already for $a \leq 1 / 3$ the theorem is not true as the following result shows.

THEOREM 1.6. For every $a, 0<a \leq 1 / 3$ there exists a complete simply connected surface $X$ with curvature $K \leq-1$ which contains an a-separated subset $\Omega \subset X$ such that every geodesic ray $\gamma:[0, \infty) \rightarrow X$ passes arbitrarily close to points of $\Omega$.

We give examples of singular as well as $C^{\infty}$-smooth surfaces $X$ satisfying Theorem 1.6. The smooth example is considerably more complicated than the singular one.

As a general reference for non-positively curved spaces we recommend for instance $[\mathbf{B a}]$.

2. Geodesics avoiding open subsets in hyperbolic space

In this section we prove Theorems 1.2, 1.4 and the result of Burns and Pollicott (Theorem 1.1) without the non-recurrence condition on $v_{0}$. We start with a theorem on the existence of geodesic rays (instead of complete geodesics) which stay away from the $\varepsilon$-neighborhood of a discrete subset. Our result is a generalized version of Theorem 1.4.

THEOREM 2.1. Let $a>0, \ell \in \mathbb{N}$ and $b \geq 1$ be given. Then there exists $\varepsilon=\varepsilon(a, \ell, b)>0$ such that the following holds. Let $X$ be a complete simply connected surface with curvature $-b^{2} \leq K \leq-1$ and $\Omega \subset X$ a subset with the properties:

(1) $d(o, \Omega) \geq a / 2$ for a distinguished point $o \in X$,

(2) for all $x \in X$ we have $\left|B_{a}(x) \cap \Omega\right| \leq \ell$, where $B_{a}(x)$ denotes the ball of radius a around $x$.

Then there exists a ray $\gamma:[0, \infty) \rightarrow X$ with $d(\gamma([0, \infty)), \Omega) \geq \varepsilon$ and $\gamma(0)=o$.

Proof. We choose geodesic polar coordinates $[0, \infty) \times S^{1}$ around $o \in X$. For an interval $I \subset S^{1}$ we use the notation $L(t, I)$ for the length of the spherical interval $(t, I) \subset X$.

We need the following estimate.

LEMMA 2.1. Let $I \subset S^{1}$ be a closed interval, $s$ and $T$ real numbers such that $L(s+$ $T, I) \leq a$. Then

$$
|\Omega \cap([s, s+T] \times I)| \leq\left(\left\lceil\frac{T}{a}\right\rceil+2\right) \cdot \ell,
$$

where \lceil\rceil denotes the integer part.

Proof. Let $\mu:=\lceil T / a\rceil+1$ and choose $x_{v} \in I$ such that $p_{v}=\left(s+v \cdot T / \mu, x_{v}\right)$ is the midpoint of the spherical interval $(s+v \cdot T / \mu, I)$ for $v=0, \ldots, \mu$. An easy calculation shows that

$$
[s, s+T] \times I \subset \bigcup_{v=0}^{\mu} B_{a}\left(p_{v}\right),
$$

thus by assumption (2) of Theorem 2.1 the lemma follows. 
On the set $\left\{(t, I) \mid t \geq a / 3, I \subset S^{1}\right\}$ of spherical intervals, we define a dilatation function at the time $T$ as

$$
\operatorname{Dil}_{T}(t, I)=\frac{L(t+T, I)}{L(t, I)} .
$$

LEMMA 2.2. There exist functions $f=f(b, T), g=g(b, T)$ such that

$$
g(b, T) \leq \operatorname{Dil}_{T}(t, I) \leq f(b, T)
$$

for all $T>0$ and all spherical intervals $(t, I)$, and $g(b, T) / T \rightarrow \infty$ as $T \rightarrow \infty$.

Proof. By the standard comparison theorems, one can take

$$
f(b, T)=c_{0} \exp (b T), \quad g(b, T)=c_{1} \exp (T)
$$

for appropriate constants $c_{0}, c_{1}>0$, which depend only on $b$.

We now fix a constant $T>0$ with $g(b, T) \geq 3 m$, where $m>2(\lceil T / a\rceil+2) \cdot \ell$, and an $\eta$ with $\eta \cdot f(b, T) \leq a$. Let $t_{-1}=0$ and $t_{n}=a / 3+n T$ for $n \geq 0$. We construct a sequence $I_{0} \supset I_{1} \supset I_{2} \supset \cdots$ of closed intervals in $S^{1}$ such that the following properties hold for all $n \geq 0$ :

(i) $L\left(t_{n}, I_{n}\right)=3 \eta$,

(ii) $\left(t_{n}, I_{n+1}\right)$ is contained in the middle third of $\left(t_{n}, I_{n}\right)$,

(iii) $\left(\left[t_{n-1}, t_{n}\right] \times I_{n}\right) \cap \Omega=\emptyset$.

Choose first an arbitrary interval $I_{0}$ such that $L\left(t_{0}, I_{0}\right)=3 \eta$. Clearly $\left(\left[t_{-1}, t_{0}\right] \times I_{0}\right) \cap$ $\Omega=\emptyset$ by assumption (1) on $\Omega$. Assume, inductively, that the interval $I_{n}$ has already been constructed and choose $I_{n}^{\prime} \subset I_{n}$ so that $\left(t_{n}, I_{n}^{\prime}\right)$ is the middle third subinterval of $\left(t_{n}, I_{n}\right)$. During the time $T$ between $t_{n}$ and $t_{n+1}$ the interval $\left(t_{n}, I_{n}^{\prime}\right)$ expands to the size

$$
L\left(t_{n+1}, I_{n}^{\prime}\right)=\operatorname{Dil}_{T}\left(t_{n}, I_{n}^{\prime}\right) L\left(t_{n}, I_{n}^{\prime}\right) \leq f(b, T) \cdot \eta \leq a .
$$

Next we divide $\left(t_{n}, I_{n}^{\prime}\right)$ into $m$ subintervals all with the same length $\eta / m$. Using Lemma 2.1 we have $\left(\left[t_{n}, t_{n+1}\right] \times I_{n}^{\prime \prime}\right) \cap \Omega=\emptyset$ for one of them, $I_{n}^{\prime \prime}$. During the time $T$ it expands to the size

$$
L\left(t_{n+1}, I_{n}^{\prime \prime}\right)=\operatorname{Dil}_{T}\left(t_{n}, I_{n}^{\prime \prime}\right) L\left(t_{n}, I_{n}^{\prime \prime}\right) \geq g(b, T) \cdot \eta / m \geq 3 \eta .
$$

Now choose $I_{n+1} \subset I_{n}^{\prime \prime}$ so that $L\left(t_{n+1}, I_{n+1}\right)=3 \eta$. It is clear that $I_{n+1}$ has the desired properties.

Let now $v \in S^{1}$ be a vector from $\cap_{n \geq 0} I_{n}$. We claim that $\gamma_{v}(t)=(t, v)$ satisfies the required properties. Suppose $(t, w) \in \Omega$. Choose $n \in \mathbb{N}$ such that $t_{n-1} \leq t \leq t_{n}$. We have by construction that

$$
(t, v) \in\left[t_{n-1}, t_{n}\right] \times I_{n+1} \subset\left[t_{n-1}, t_{n}\right] \times I_{n}
$$

and $(t, w) \notin\left[t_{n-1}, t_{n}\right] \times I_{n}$ by (iii). It follows from (ii) that the distance between $\gamma_{v}(t)$ and $(t, w)$ measured on the distance sphere of radius $t$ is bounded below by

$$
\frac{L\left(t_{n}, I_{n}\right)}{3 \cdot f\left(b, t_{n}-t\right)}=\frac{\eta}{f\left(b, t_{n}-t\right)}
$$

Note that $0 \leq t_{n}-t \leq T$ and $T$ depends only on $a$, $\ell$ and $b$. Hence there exists $\varepsilon=\varepsilon(a, \ell, b)>0$ with $d\left(\gamma_{v}([0, \infty)),(t, w)\right) \geq \varepsilon$ for all $(t, w) \in \Omega$. 
The symmetry of $\mathbb{H}^{2}$ allows us to get a version for complete geodesics.

THEOREM 2.2. Let $X$ be the hyperbolic plane $\mathbb{H}^{2}$. For $a>0$ and $\ell \in \mathbb{N}$ there exists $\varepsilon_{1}=\varepsilon_{1}(a, \ell)>0$ such that for any set $\Omega$ as in Theorem 2.1 there exists a complete geodesic $\gamma: \mathbb{R} \rightarrow \mathbb{H}^{2}, \gamma(0)=o$, such that $d(\gamma(\mathbb{R}), \Omega) \geq \varepsilon_{1}$.

Proof. Let $\sigma: \mathbb{H}^{2} \rightarrow \mathbb{H}^{2}$ be the reflection at the origin $o$. Define $\Omega^{\prime}:=\Omega \cup \sigma(\Omega)$. Then $\sigma\left(\Omega^{\prime}\right)=\Omega^{\prime}$. Since $\sigma$ is an isometry, $\Omega^{\prime}$ satisfies the properties:

$\left(1^{\prime}\right) \quad d\left(o, \Omega^{\prime}\right) \geq a$,

(2') for all $x \in \mathbb{H}^{2}$ we have $\left|B_{a}(x) \cap \Omega^{\prime}\right| \leq 2 \ell$.

By Theorem 2.1 there exists a ray $\gamma:[0, \infty) \rightarrow \mathbb{H}^{2}$ with $\gamma(0)=o$ and $d\left(\gamma([0, \infty)), \Omega^{\prime}\right) \geq \varepsilon_{1}=\varepsilon(a, 2 \ell)$. Let $\gamma$ also denote the complete extension $\gamma: \mathbb{R} \rightarrow \mathbb{H}^{2}$, then $\gamma(\mathbb{R})=\gamma([0, \infty)) \cup \sigma(\gamma([0, \infty)))$. Thus by the $\sigma$-invariance of $\Omega^{\prime}$ we conclude that $d\left(\gamma(\mathbb{R}), \Omega^{\prime}\right) \geq \varepsilon_{1}$.

An easy modification of the proofs of Theorems 2.1 and 2.2, allows us to choose the initial vector $\dot{\gamma}(0)$ in a given interval. To get the following result one has merely to choose the 'initial' interval $I_{0}$ in the proof of Theorem 2.1 appropriately.

THEOREM 2.3. Let $X$ be the hyperbolic plane $\mathbb{H}^{2}$. For given $a>0, \ell \in \mathbb{N}$ and $\eta>0$ there exists $\varepsilon_{1}(a, \ell, \eta)>0$ such that for any set $\Omega$ as in Theorem 2.1 and any $\bar{w} \in S_{o} \mathbb{H}^{2}$ there exists a complete geodesic $\gamma: \mathbb{R} \rightarrow \mathbb{H}^{2}, \gamma(0)=o$, such that $d(\gamma(\mathbb{R}), \Omega) \geq \varepsilon_{1}$ and $d^{1}(\dot{\gamma}(0), \bar{w}) \leq \eta$.

We are now able to prove the result of Burns and Pollicott without the non-recurrence condition on $v_{0}$.

THEOREM 2.4. Let $M=\Gamma \backslash \mathbb{H}^{2}$ be the quotient of $\mathbb{H}^{2}$ by a discrete torsion-free group $\Gamma$ and let $v_{0} \in S M$ be arbitrary. Then there exists $\varepsilon>0$ and for every point $x \in M$ a unit speed geodesic $\gamma_{x}: \mathbb{R} \rightarrow M$ with $\gamma_{x}(0)=x$ such that $d^{1}\left(\dot{\gamma}(t), v_{0}\right) \geq \varepsilon$ for all $t \in \mathbb{R}$.

Proof. Let $p: \mathbb{H}^{2} \rightarrow M$ be the universal covering. Let $\Omega^{*}:=p^{-1}\left(\pi\left(v_{0}\right)\right)$ and $\bar{x}$ a point in $p^{-1}(x)$. There exists $a>0$ such that for distinct points $q_{1}, q_{2} \in \Omega^{*}$ the condition $d\left(q_{1}, q_{2}\right)>2 a$ holds.

We first assume that $B_{a}(\bar{x}) \cap \Omega^{*}=\emptyset$. Then by Theorem 2.2 there exists a geodesic $\gamma: \mathbb{R} \rightarrow \mathbb{H}^{2}, \gamma(0)=\bar{x}$ with $d(\gamma(\mathbb{R}), \Omega) \geq \varepsilon_{1}$. Obviously, $\gamma_{x}:=p \circ \gamma$ satisfies the required conditions.

Let us finally consider the case $B_{a}(\bar{x}) \cap \Omega^{*} \neq \emptyset$. Since $d\left(q_{1}, q_{2}\right)>2 a$ for any two distinct points in $\Omega^{*}$, we see that $B_{a}(\bar{x}) \cap \Omega^{*}$ consists of only one point $\bar{y}$. Let $\bar{v}_{0} \in S_{\bar{y}} \mathbb{H}^{2}$ be the vector with $p_{*, \bar{y}}\left(\bar{v}_{0}\right)=v_{0}$, where $p_{*, \bar{y}}$ is the differential of the map $p$ at the point $\bar{y} \in \mathbb{H}^{2}$. The following result is rather obvious and not difficult to prove.

LEMMA 2.3. There exists $\varepsilon_{2}>0$ such that for every $\bar{x} \in \mathbb{H}^{2}$ and every $\bar{v}_{0} \in S \mathbb{H}^{2}$ there is a vector $\bar{w} \in S_{\bar{x}} \mathbb{H}^{2}$ with the following property.

For all $\bar{u} \in S_{\bar{x}} \mathbb{H}^{2}$ with $d^{1}(\bar{u}, \bar{w})<\varepsilon_{2}$ we have $d^{1}\left(\dot{\gamma}_{\bar{u}}(t), \bar{v}_{0}\right) \geq \pi / 4$ for all $t \in \mathbb{R}$. Here $\gamma_{\bar{u}}$ is the geodesic with $\dot{\gamma}_{\bar{u}}(0)=\bar{u}$.

Now choose $\bar{w}$ as in Lemma 2.3 and let $\Omega:=\Omega^{*} \backslash\{\bar{y}\}$. By Theorem 2.3 there exists $\varepsilon_{1}=\varepsilon_{1}\left(a, 1, \varepsilon_{2}\right)>0$ such that there is a complete geodesic $\gamma: \mathbb{R} \rightarrow \mathbb{H}^{2}$ 


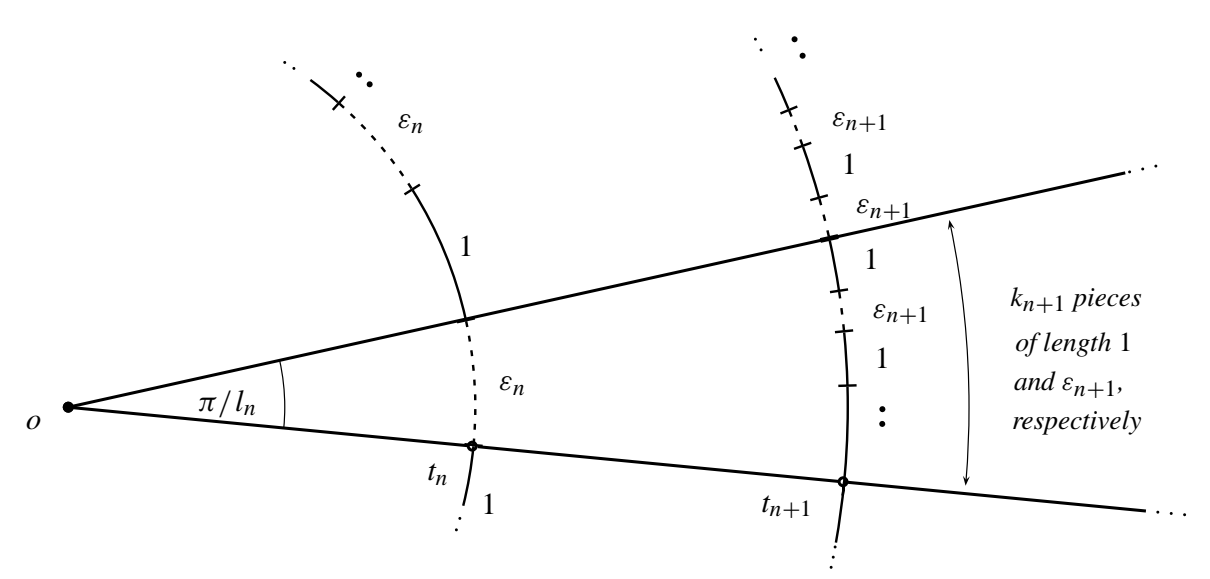

FIGURE 1. Construction of the radii $t_{n}$.

with $\gamma(0)=\bar{x}, d(\gamma(\mathbb{R}), \Omega) \geq \varepsilon_{1}$ and $d^{1}(\dot{\gamma}(0), \bar{w}) \leq \varepsilon_{2}$. By Lemma 2.3 we infer that $d^{1}\left(\dot{\gamma}(t), \bar{v}_{0}\right) \geq \pi / 4$ for all $t \in \mathbb{R}$. Therefore, the geodesic $\gamma_{x}=p \circ \gamma: \mathbb{R} \rightarrow M$ satisfies $d^{1}\left(\dot{\gamma}_{x}(t), v_{0}\right) \geq \min \left\{\varepsilon_{1}, \pi / 4\right\}$ for all $t \in \mathbb{R}$.

\section{Proof of Theorem 1.3}

In this section we construct an example which proves Theorem 1.3. We first give an outline of the construction and later we fill in the necessary details.

We write the space $\mathbb{R}^{2}$ in planar polar coordinates as $[0, \infty) \times S^{1}$ and choose a monotonically decreasing null sequence $\left(\varepsilon_{n}\right)_{n \in \mathbb{N}}$ with $\varepsilon_{1}=1$. In an inductive process, which we describe more precisely below, we construct an increasing sequence $0<$ $t_{1}<t_{2}<\cdots$ of 'radii' and a sequence $\left(k_{n}\right)_{n \in \mathbb{N}}$ of odd integers. Then we divide the plane into sectors $S_{n, v}=\left[0, t_{n}\right] \times\left[(v-1) \cdot \pi / l_{n}, v \cdot \pi / l_{n}\right], v=1, \ldots, 2 l_{n}$, with $l_{n}:=k_{1} \cdot k_{2} \cdots k_{n}$. Note that $l_{n}$ is odd for all $n \in \mathbb{N}$. We will define (warped product) metrics $g_{n, v}$ on $S_{n, v}$ of the form $d t^{2}+f_{n, v}^{2}(t) d \theta^{2}, v=1, \ldots, 2 l_{n}$, where the warping function $f_{n, v}:\left[0, t_{n}\right] \rightarrow[0, \infty)$ is a positive smooth function such that $f_{n, v}(0)=0$, $\dot{f}_{n, v}(0)=1$ and $\ddot{f}_{n, v}(t) \geq 0$ for all $t$. The functions will be chosen such that the sectional curvature satisfies $K_{g_{n}} \mid S_{n, v}=-\ddot{f}_{n, v} / f_{n, v} \in[-1-\delta,-1+\delta]$. This choice for the metric implies, in particular, that we have rotational symmetry within a sector $S_{n, v}$.

Let $C_{n}$ be the circle of radius $t_{n}$ around the origin. This circle is divided into the intervals $C_{n, v}:=\left\{t_{n}\right\} \times\left[(v-1) \cdot \pi / l_{n}, v \cdot \pi / l_{n}\right], v=1, \ldots, 2 l_{n}$. We will choose the metric $g$ in such a way that $C_{n, v}$ has length $\varepsilon_{n}$ for $v$ odd and length 1 for $v$ even. Then we choose $\Omega_{n}$ to be the set of the midpoints $p_{n, v}:=\left(t_{n},(2 v-1) \cdot \pi / 2 l_{n}\right), v=1,3, \ldots, 2 l_{n}-1$, of the odd intervals $C_{n, \nu}$. Thus, the concentric circles $C_{n}$ are alternately divided into two types of intervals, namely intervals of length $\varepsilon_{n}$ (called small intervals) and intervals of length 1 (called large intervals). Finally, we put $\Omega=\cup_{n \geq 1} \Omega_{n}$.

Let us assume that we have constructed a metric $g$ with these properties. Consider a unit 
speed geodesic $\gamma: \mathbb{R} \rightarrow\left(\mathbb{R}^{2}, g\right), \gamma(0)=o$. Note that on the one hand, if $\gamma\left(t_{n}\right) \in C_{n, v}$ and $v \in\left\{1, \ldots, l_{n}\right\}$ then $\gamma\left(-t_{n}\right) \in C_{n, l_{n}+v}$ and, on the other hand, if $\gamma\left(t_{n}\right) \in C_{n, v}$ and $v \in\left\{l_{n}+1, \ldots, 2 l_{n}\right\}$ then $\gamma\left(-t_{n}\right) \in C_{n,-l_{n}+v}$. Since we have $2 l_{n} \equiv 2 \bmod 4$ by construction, $\gamma\left(t_{n}\right)$ lies in a small interval if and only if $\gamma\left(-t_{n}\right)$ lies in a large interval.

We show that this implies that $d(\gamma(\mathbb{R}), \Omega)=0$. Assume that $d(\gamma([0, \infty)), \Omega) \geq \eta>0$ and let $n$ be so large that $\varepsilon_{n}<2 \eta$. Since $d\left(\gamma\left(t_{n}\right), \Omega\right) \geq \varepsilon_{n} / 2$, the point $\gamma\left(t_{n}\right)$ is contained in a large interval, thus $\gamma\left(-t_{n}\right)$ is contained in a small interval. Hence $d\left(\gamma\left(-t_{n}\right), \Omega\right) \leq \varepsilon_{n}$ for all $n$ large enough and therefore $d(\gamma(\mathbb{R}), \Omega)=0$ as claimed.

We now fill in the details of the construction. We are given the sequence $\left(\varepsilon_{n}\right)_{n \in \mathbb{N}}$, and we have to construct inductively $t_{n}, k_{n}$ and the warping functions $f_{n, v}, v=1, \ldots, 2 l_{n}$, where $l_{n}=\prod_{i=1}^{n} k_{i}$. The condition on the length of the intervals $C_{n, v}$ corresponds to the condition that

and

$$
f_{n, v}\left(t_{n}\right)=\frac{l_{n}}{\pi} \cdot \varepsilon_{n}, \quad \text { for } v \text { odd }
$$

$$
f_{n, v}\left(t_{n}\right)=\frac{l_{n}}{\pi} \cdot 1, \quad \text { for } v \text { even. }
$$

Note that $\varepsilon_{1}=1$. This enables us to choose the metric to be the standard hyperbolic metric near the origin. We may choose for example $k_{1}=3, t_{1}=\operatorname{arcsinh}(3 / \pi)$ and $f_{1, v}(t)=\sinh (t), v=1, \ldots, 6$.

Let us assume that we have already constructed $k_{n}, t_{n}$ and $f_{n, v}, v=1, \ldots, 2 l_{n}$. In the next step we have to construct $k_{n+1}, t_{n+1}$ and $f_{n+1, v}$. In order to do so we need the following extension lemma and its corollary.

LEMMA 3.1. Let $t_{a}$ be a positive real number and $f:\left[0, t_{a}\right] \rightarrow[0, \infty)$ be a non-negative smooth function satisfying the differential inequality

$$
-1-\delta \leq-\frac{\ddot{f}(t)}{f(t)} \leq-1+\delta, \quad \text { for some constant } \delta \in(0,1),
$$

for all $t \in\left(0, t_{a}\right]$ together with the initial conditions

$$
f(0)=0 \quad \text { and } \quad \dot{f}(0)=1 .
$$

Let $\sinh _{+}(t):=\sinh \left(\sqrt{1+\delta_{1}} t\right)$ and $\sinh _{-}(t):=\sinh \left(\sqrt{1-\delta_{1}} t\right)$, where $\delta_{1}$ is some constant in $(0, \delta)$. Consider the family $\mathcal{F}_{f}$ of $C^{\infty}$ extensions of $f, \mathcal{F}_{f}=\{\bar{f}:[0, \infty) \rightarrow$ $[0, \infty) \mid \bar{f}$ is a $C^{\infty}$ function satisfying (3.0.1) for all $t \in(0, \infty)$ and such that $\left.\left.\bar{f}\right|_{\left[0, t_{a}\right]}=f\right\}$.

Moreover, let

$$
\mathcal{F}_{f}(t):=\left\{g(t) \mid g \in \mathcal{F}_{f}\right\}
$$

be the slices of $\mathcal{F}_{f}$.

Then there exists a real number $t_{b}=t_{b}(f)>t_{a}$ depending on the given function $f$ such that

$$
\left[\sinh _{-}(t), \sinh _{+}(t)\right] \subset \mathcal{F}_{f}(t), \quad \forall t \geq t_{b}
$$


Proof of Lemma 3.1. Consider the $C^{\infty}$ function $\varphi:\left[0, t_{a}\right] \rightarrow[1-\delta, 1+\delta]$ defined by the differential equation $\varphi=\ddot{f} / f$ (i.e. $\varphi(t)$ is the modulus of the sectional curvature at $\left.t \in\left[0, t_{a}\right]\right)$. Let $\bar{\varphi}:[0, \infty) \rightarrow[1-\delta, 1+\delta]$ be an extension of $\varphi$. Then the solution of the differential equation $\ddot{\bar{f}} / \bar{f}=\bar{\varphi}$ with initial conditions $\bar{f}(0)=0$ and $\dot{\bar{f}}(0)=1$ is an extension of $f$, hence $\bar{f} \in \mathcal{F}_{f}$.

Let $\bar{\varphi}_{0}, \bar{\varphi}_{1}:[0, \infty) \rightarrow[1-\delta, 1+\delta]$ be $C^{\infty}$ functions with $\left.\bar{\varphi}_{0}\right|_{\left[0, t_{a}\right]}=\varphi=\left.\bar{\varphi}_{1}\right|_{\left[0, t_{a}\right]}$ and $\left.\bar{\varphi}_{0}\right|_{\left[t_{a}+1, \infty\right)} \equiv 1-\delta,\left.\bar{\varphi}_{1}\right|_{\left[t_{a}+1, \infty\right)} \equiv 1+\delta$. Let $\bar{\varphi}_{s}:=s \bar{\varphi}_{0}+(1-s) \bar{\varphi}_{1}, s \in[0,1]$, and let the solutions of $\ddot{\bar{f}}_{s} / \bar{f}_{s}=\bar{\varphi}_{s}, \bar{f}_{s} \in \mathcal{F}_{f}$, be $C^{\infty}$ extensions of $f$. Note that $\left.\bar{f}_{1}\right|_{\left[t_{a}+1, \infty\right)}(t)=A_{1} \sinh (\sqrt{1+\delta} t)+B_{1} \cosh (\sqrt{1+\delta} t)$, where $A_{1}+B_{1}>0$, since $\left.\bar{f}_{1}\right|_{(0, \infty)}$ is positive and convex. Thus $\bar{f}_{1}(t)>\sinh _{+}(t)$ for $t$ large enough because of $\delta>\delta_{1}$. Similarly, $\bar{f}_{0}(t)<\sinh _{-}(t)$ for $t$ sufficiently large. Hence, we conclude by the continuous dependence of the $\bar{f}_{s}$ on the parameter $s$ that $\left\{\bar{f}_{s}(t) \mid s \in[0,1]\right\} \supset$ $\left[\sinh _{-}(t), \sinh _{+}(t)\right]$ for $t$ sufficiently large.

It is not difficult to generalize Lemma 3.1 to finitely many functions.

COROLlaRY 3.1. Let $t_{a}$ be a positive real number and $f_{1}, \ldots, f_{N}:\left[0, t_{a}\right] \rightarrow[0, \infty)$ be non-negative convex $C^{\infty}$ functions satisfying the differential inequalities

$$
-1-\delta \leq-\frac{\ddot{f}_{i}(t)}{f_{i}(t)} \leq-1+\delta, \quad i \in\{1, \ldots, N\},
$$

for all $t \in\left(0, t_{a}\right]$ together with the initial conditions $f_{i}(0)=0$ and $\dot{f}_{i}(0)=1$ for all $i \in\{1, \ldots, N\}$.

Then there exists a real number $t_{b}=t_{b}\left(f_{1}, \ldots, f_{N}\right)>t_{a}$ depending on the given functions $f_{1}, \ldots, f_{N}$ such that for any given $\tau \geq t_{b}$ and arbitrary $s_{i} \in\left[\sinh _{-}(t), \sinh _{+}(t)\right]$ there exist extensions $\bar{f}_{i} \in \mathcal{F}_{f_{i}}$ with $\bar{f}_{i}(\tau)=s_{i}$ for all $i \in\{1, \ldots, N\}$.

We now complete the induction step. We will apply Corollary 3.1 to the functions $f_{n, v}$, $v=1, \ldots, 2 l_{n}$, for $t_{a}=t_{n}$. Now choose $t_{n+1}$ such that

$$
t_{n+1} \geq t_{b}\left(f_{n, 1}, \ldots, f_{n, 2 l_{n}}\right)
$$

and in addition

$$
\frac{\sinh _{+}\left(t_{n+1}\right)-\sinh _{-}\left(t_{n+1}\right)}{\sinh _{-}\left(t_{n+1}\right)} \geq \frac{3}{\varepsilon_{n+1}} .
$$

Next we choose $k_{n+1}$ to be the largest odd number such that

$$
\frac{1}{\pi} \cdot k_{n+1} \cdot l_{n}=\frac{1}{\pi} \cdot l_{n+1} \leq \sinh _{+}\left(t_{n+1}\right) .
$$

It remains to construct $f_{n+1, v}$ for $v=1, \ldots, 2 l_{n+1}$. Fix $v \in\left\{1, \ldots, 2 l_{n+1}\right\}$ and let $\mu \in\left\{1, \ldots, 2 l_{n}\right\}$ be the number such that $S_{n+1, v} \subset S_{n, \mu}$. In order that the metric on $\left[0, t_{n+1}\right] \times S^{1}$ is an extension of the metric on $\left[0, t_{n}\right] \times S^{1}$, the new corresponding warping function $f_{n+1, v}$ has to be an extension of $f_{n, \mu}$. To have the required length condition on the interval $C_{n+1, v}$ we have to choose $f_{n+1, v}\left(t_{n+1}\right)=\left(l_{n+1} / \pi\right) \cdot \varepsilon_{n+1}$ for $v$ odd and $f_{n+1, v}\left(t_{n+1}\right)=\left(l_{n+1} / \pi\right) \cdot 1$ for $v$ even.

By (3.0.3) and Corollary 3.1 the existence of these extensions is guaranteed if we show that $\left[\varepsilon_{n+1} \cdot\left(l_{n+1} / \pi\right),\left(l_{n+1} / \pi\right)\right] \subset\left[\sinh _{-}\left(t_{n+1}\right), \sinh _{+}\left(t_{n+1}\right)\right]$. Now inequality (3.0.5) 
implies that $l_{n+1} / \pi \leq \sinh _{+}\left(t_{n+1}\right)$. Since $k_{n+1}$ is the largest odd number satisfying (3.0.5), we have

$$
\frac{1}{\pi} \cdot\left(k_{n+1}+2\right) \cdot l_{n}>\sinh _{+}\left(t_{n+1}\right) .
$$

Using (3.0.4) we obtain

$$
\sinh _{+}\left(t_{n+1}\right) \geq\left(\frac{3}{\varepsilon_{n+1}}+1\right) \sinh _{-}\left(t_{n+1}\right)>\frac{3}{\varepsilon_{n+1}} \sinh _{-}\left(t_{n+1}\right) .
$$

Because of $l_{n}<l_{n+1}$ we get from (3.0.6) and (3.0.7) that

$$
\begin{aligned}
\sinh _{-}\left(t_{n+1}\right) & <\frac{\varepsilon_{n+1}}{3} \cdot\left(k_{n+1}+2\right) \cdot \frac{l_{n}}{\pi}=\frac{\varepsilon_{n+1}}{3} \cdot \frac{l_{n+1}}{\pi}+\frac{2 \varepsilon_{n+1}}{3} \cdot \frac{l_{n}}{\pi} \\
& <\varepsilon_{n+1} \cdot \frac{l_{n+1}}{\pi} .
\end{aligned}
$$

The metric constructed so far is not smooth. So finally we indicate how to make it smooth. This is done in two steps. In the first step we modify the construction in such a way that on a sequence $\left(\left[s_{n}, s_{n}+1\right] \times S^{1}\right)_{n \in \mathbb{N}}$ of annuli the metric coincides with the hyperbolic metric. By standard methods one proves the following.

LEMMA 3.2. Let $f:\left[0, t_{a}\right] \rightarrow[0, \infty)$ be as in Lemma 3.1. Then there exist a number $t_{c}=t_{c}(f)>t_{a}$ and a function $\bar{f} \in \mathcal{F}_{f}$ with $\left.\bar{f}\right|_{\left[t_{c}, \infty\right)}(t)=\sinh (t)$.

Combining Lemma 3.2 with Corollary 3.1 one can obtain the following corollary.

Corollary 3.2. Let $f_{1}, \ldots, f_{N}:\left[0, t_{a}\right] \rightarrow[0, \infty)$ be as in Corollary 3.1. Then there exist constants $t_{b}\left(f_{1}, \ldots, f_{N}\right)$ and $t_{c}\left(f_{1}, \ldots, f_{N}\right)$ with $t_{b}>t_{c}+1>t_{c}>t_{a}$ such that for any given $\tau \geq t_{b}$ and all $s_{i} \in\left[\sinh _{-}(t), \sinh _{+}(t)\right], i=1, \ldots, N$, there are extensions $\bar{f}_{i} \in \mathcal{F}_{f_{i}}$ with $\bar{f}_{i}(\tau)=s_{i}$ for all $i \in\{1, \ldots, N\}$ and $\left.\bar{f}_{i}\right|_{\left[t_{c}, t_{c}+1\right]}=\left.\sinh \right|_{\left[t_{c}, t_{c}+1\right]}$.

If we use the extension in this way, the metric is not smooth on a locally finite set of slits of the form $[\alpha, \beta] \times v$ with real numbers $\alpha<\beta$. We now show how to smooth the metric on these slits. We identify $v$ with $0 \in S^{1}$. In a small box $(\alpha-\varepsilon, \beta+\varepsilon) \times(-\varepsilon, \varepsilon)$, $\varepsilon>0$, the metric is of the form

$$
\begin{array}{ll}
d t^{2}+f_{+}^{2}(t) \cdot d \theta^{2}, & \text { for } \theta \geq 0 \\
d t^{2}+f_{-}^{2}(t) \cdot d \theta^{2}, & \text { for } \theta \leq 0
\end{array}
$$

and $f_{+}(t)=f_{-}(t)=\sinh (t)$ for $t \in(\alpha-\varepsilon, \alpha+\varepsilon)$ and $t \in(\beta-\varepsilon, \beta+\varepsilon)$.

We take a suitable partition of unity $\lambda, 1-\lambda$ on $(-\varepsilon, \varepsilon)$ and consider the metric

$$
d t^{2}+\left[\lambda(\theta) \cdot f_{+}(t)+(1-\lambda(\theta)) \cdot f_{-}(t)\right]^{2} \cdot d \theta^{2}
$$

Note that the new metric is smooth and coincides with the old metric near the boundary of the box. Moreover, an easy calculation shows that the curvature satisfies the same pinching and the lines $[\alpha, \beta] \times \theta$ are still geodesics. Thus we obtain a smooth metric with the same properties as the non-smooth one. 


\section{Proof of Theorem 1.5}

In $\S 2$ we proved Theorem 1.4, which is a generalization of the result of Burns and Pollicott. There we developed a different approach to avoid the lower bound on the separation number $a$ which is essential in the argument of $[\mathbf{B P}]$. The original argument in $[\mathbf{B P}]$, however, has the advantage that it does not need the lower curvature bound and hence is also valid in the context of singular spaces.

In this section we elaborate these methods and prove Theorem 1.5. While the idea of the argument is clear, the proof itself is technical since we want to obtain an 'optimal' lower bound for the separation constant. The bound $\ln 2$ seems to be optimal for this type of argument, and the example of Theorem 1.6 shows that it is close to the optimal constant.

We briefly sketch the idea of the argument. We call an $\varepsilon$-ball around a point $\omega \in \Omega$ an 'obstacle'. We start with an arbitrary ray from $o$ and follow this ray until we hit the first obstacle. Then we modify the ray so that we avoid the obstacle, and we follow the modified ray until we hit the next obstacle. The main point is to show that the sum of all later modifications does not destroy what we have done in the first steps.

We prove the result in the following setting.

THEOREM 4.1. For every $a_{0}>\ln 2$ there exists an $\varepsilon_{0}>0$ with the following property.

Let $X$ be a complete $C A T(-1)$-space with extendable geodesics such that the space of directions at every point contains at least three elements, and let $\Omega \subset X$ be an $a_{0}$-separated subset. Then for each $o \in X, d(o, \Omega) \geq a_{0} / 2$ there exists a geodesic ray $\gamma:[0, \infty) \rightarrow X$ with $d(\gamma([0, \infty)), \Omega) \geq \varepsilon_{0}$ and $\gamma(0)=o$.

4.1. The choice of constants. We fix $a_{1}$ with $\ln 2<a_{1}<a_{0}$ and choose $a \in\left(\ln 2, a_{1}\right)$, $\varepsilon>0$ such that the following conditions hold:

$$
\begin{gathered}
e^{a_{1}-a}<e^{a_{1}}-1 ; \\
2(\cosh \varepsilon-1) \leq \varepsilon^{2} e^{2\left(a_{1}-a\right)} ; \\
a_{1}+10 \varepsilon \leq a_{0} .
\end{gathered}
$$

4.2. An estimate for triangles in $\mathbb{H}^{2}$. For $T>0, n \in \mathbb{N}$ let $\Delta_{T, n} \subset \mathbb{H}^{2}$ be an isosceles triangle, $\Delta_{T, n}=o x_{T}^{\prime} y_{T}^{\prime}, d\left(o, x_{T}^{\prime}\right)=d\left(o, y_{T}^{\prime}\right)=T+n a_{1}$ with the base length $d\left(x_{T}^{\prime}, y_{T}^{\prime}\right)=\varepsilon$. We put $z_{n}(T)=d\left(x_{T}, y_{T}\right)$, where the points $x_{T} \in o x_{T}^{\prime}, y_{T} \in o y_{T}^{\prime}$ satisfy $d\left(o, x_{T}\right)=d\left(o, y_{T}\right)=T$.

LEMMA 4.1. For each $T>0, n \geq 0$ we have

$$
z_{n+1}(T)<\varepsilon e^{-\left(a+n a_{1}\right)} \text {. }
$$

Proof. The function $z_{n}=z_{n}(T)$ strictly increases on $(0, \infty)$ as it is easy to see if one puts together the bases of corresponding triangles $\Delta_{T, n}, \Delta_{T^{\prime}, n}$. Using formulas of hyperbolic trigonometry, we obtain

$$
\cosh z_{n}(T)=\cosh ^{2}(T)-\sinh ^{2}(T) \cos \phi,
$$

where $\phi=L_{o}\left(x_{T}, y_{T}\right)$. On the other hand,

$$
\cosh \varepsilon=\cosh ^{2}\left(T+n a_{1}\right)-\sinh ^{2}\left(T+n a_{1}\right) \cos \phi,
$$


thus

$$
\cosh z_{n}(T)=\cosh ^{2}(T)-\sinh ^{2}(T) \frac{\cosh ^{2}\left(T+n a_{1}\right)-\cosh \varepsilon}{\sinh ^{2}\left(T+n a_{1}\right)} .
$$

A simple calculation now shows that

$$
\lim _{T \rightarrow \infty} \cosh z_{n}(T)=1+(\cosh \varepsilon-1) e^{-2 n a_{1}} .
$$

Therefore, $\cosh z_{n}(T)-1<(\cosh \varepsilon-1) e^{-2 n a_{1}}$ for all $T>0$. By (4.1.2) we have

$$
\frac{z_{n}^{2}(T)}{2}<\cosh z_{n}(T)-1<\frac{\varepsilon^{2}}{2} e^{-2\left(a+(n-1) a_{1}\right)} .
$$

Hence

$$
z_{n}(T)<\varepsilon e^{-\left(a+(n-1) a_{1}\right)}
$$

4.3. The construction of a required geodesic ray $\gamma$. We start with the following construction of a geodesic ray $\gamma$ with the required properties.

Lemma 4.2. Assume that $o, \omega \in X$ with $d(o, \omega)>5 \varepsilon$ and that a point $y \in X$, $d(y, \omega)=\varepsilon$, is the closest point of the segment oy to $\omega$. Let $x^{0} \in$ oy be the point with $d\left(y, x^{0}\right)=4 \varepsilon$. Then there exists a ray $\gamma \subset X$ starting at o with $d(\gamma, \omega) \geq \varepsilon$ such that for the point $x^{1} \in \gamma$ with $d\left(o, x^{1}\right)=d\left(o, x^{0}\right)$ we have $d\left(x^{0}, x^{1}\right)<\varepsilon$.

Proof. Let $x \in o \omega$ be the point of $o \omega$ which is closest to $x^{0}$. By convexity of the distance function we have $d\left(x^{0}, \omega\right) \geq 4 \varepsilon, d\left(x, x^{0}\right)<\varepsilon$, in particular, $d(x, \omega) \geq 3 \varepsilon$.

Assume, first, that $x \neq x^{0}$. Then there exists a $y^{1} \in X$ such that $x^{0} \in x y^{1}$ and $d\left(x^{0}, y^{1}\right)=\varepsilon$. Now let $\gamma \subset X$ be a ray outgoing from $o$, for which $y^{1} \in \gamma$. Then $x$ is the point of $o \omega$ closest to $y^{1}$ and $\varepsilon \leq d\left(x, y^{1}\right)<2 \varepsilon$. In particular, by convexity of the distance function, we have $d\left(\omega, \gamma \backslash\left[o y^{1}\right)\right) \geq \varepsilon$ and $d\left(x^{0}, x^{1}\right)<\varepsilon$ for $x^{1} \in \gamma$ with $d\left(o, x^{1}\right)=d\left(o, x^{0}\right)$.

Furthermore, the segment $o x^{\prime} \subset \gamma$ is separated from $\omega$ by the distance $>\varepsilon$ for $x^{\prime} \in \gamma$ with $d\left(o, x^{\prime}\right)=d(o, x)$, because $x \in o \omega$ and $d(x, \omega) \geq 3 \varepsilon$. Thus, if one would suppose that $d(\gamma, \omega)<\varepsilon$, then any $x^{\prime \prime} \in \gamma$ with $d\left(\omega, x^{\prime \prime}\right)<\varepsilon$ would lie between $x^{\prime}$ and $y^{1}$. For that point we would have $d\left(x^{\prime \prime}, y^{1}\right)<d\left(y^{1}, x\right)<2 \varepsilon$ and

$$
d\left(x^{0}, \omega\right) \leq d\left(x^{0}, y^{1}\right)+d\left(y^{1}, x^{\prime \prime}\right)+d\left(x^{\prime \prime}, \omega\right)<\varepsilon+2 \varepsilon+\varepsilon=4 \varepsilon .
$$

This contradicts the condition $d\left(x^{0}, \omega\right) \geq 4 \varepsilon$. Thus $d(\gamma, \omega) \geq \varepsilon$.

Assume now that $x=x^{0}$. By hypothesis, the direction space to $X$ at $x$ contains at least three elements. It follows that there exists $z \in X \backslash o \omega$ as close as we like to $x$. Hence, the arguments above are applicable to $x^{0}=z$.

We put

$$
\varepsilon_{0}:=\varepsilon\left(1-\frac{e^{a_{1}-a}}{e^{a_{1}}-1}\right)
$$


By (4.1.1) we have $\varepsilon_{0}>0$, and it follows from Lemma 4.1 that

$$
\sum_{n \geq 1} z_{n}(T)<\varepsilon-\varepsilon_{0}
$$

for each $T>0$.

For each $\omega \in \Omega$ we consider the closed ball $B_{\varepsilon}(\omega)$ of radius $\varepsilon$ around $\omega$. Using the fact that the geodesic segments in $X$ are extendable, we move from $o$ along a geodesic ray until we hit a next obstacle. Let $y_{k}$ be the meeting point with $k$ th obstacle $B_{\varepsilon}\left(\omega_{k}\right)$ which lies on a ray $\gamma_{k} \subset X$ with the initial point $o, d\left(y_{k}, \omega_{k}\right)=\varepsilon, k \geq 1$. By Lemma 4.2, there is a ray $\gamma_{k+1} \subset X$ outgoing from $o$ such that $\gamma_{k+1} \cap B_{\varepsilon}\left(\omega_{k}\right)=\emptyset$ and for $x_{k}^{1} \in \gamma_{k+1}$, $d\left(x_{k}^{1}, o\right)=d\left(x_{k}^{0}, o\right)$ the condition $d\left(x_{k}^{0}, x_{k}^{1}\right)<\varepsilon$ holds, where $x_{k}^{0} \in o y_{k}, d\left(x_{k}^{0}, y_{k}\right)=4 \varepsilon$. Then we continue our trip along $\gamma_{k+1}$.

This procedure gives the sequence $\left\{x_{k}^{0}\right\} \subset X$. We claim that the sequence of geodesic segments $o x_{k}^{0}$ converges to a ray $\gamma$ with $\gamma(0)=o$ and $d(\gamma, \Omega) \geq \varepsilon_{0}$.

To prove this, we first note that

$$
d\left(x_{k}^{1}, x_{k+1}^{0}\right) \geq a_{1}, \quad \text { for every } \quad k \geq 1 .
$$

Indeed, $d\left(x_{k}^{1}, x_{k+1}^{0}\right) \geq d\left(\omega_{k}, \omega_{k+1}\right)-d\left(x_{k}^{1}, \omega_{k}\right)-d\left(x_{k+1}^{0}, \omega_{k+1}\right) \geq a_{0}-10 \varepsilon \geq a_{1}$ by the choice (4.1.3).

Next, we consider points $x_{k-i}^{i} \in o x_{k}^{0}, i=1, \ldots, k-1$ defined by the condition $d\left(o, x_{k-i}^{i}\right)=d\left(o, x_{k-i}^{0}\right)$. It follows from (4.3.1) that $d\left(x_{k-i}^{i}, x_{k-(i-1)}^{i-1}\right) \geq a_{1}$ for each $i=1, \ldots, k-1$.

Now fix a $T>0$. There is a $k \geq 0$ such that $d\left(o, x_{k}^{0}\right)<T \leq d\left(o, x_{k+1}^{0}\right)$, where $x_{0}^{0}:=o$. Then for the point $x_{1} \in o x_{k+1}^{0}$ with $d\left(o, x_{1}\right)=T$, we have $d\left(x_{1}, \Omega\right) \geq \varepsilon$ by the construction of the segment $o x_{k+1}^{0}$. This generates a sequence of points $x_{n} \in o x_{k+n}^{0}, n \geq 1$ with $d\left(o, x_{n}\right)=T$. By the construction, we have $x_{n} \in x_{k}^{n} x_{k+1}^{n-1}$, thus $d\left(x_{n}, x_{k+n}^{0}\right) \geq$ $(n-1) a_{1}$. Recall that we also have $d\left(x_{k+n}^{0}, x_{k+n}^{1}\right)<\varepsilon$. Applying Lemma 4.1 to a comparison triangle in $\mathbb{H}^{2}$ for the triangle $o x_{k+n}^{0} x_{k+n}^{1} \subset X$, we obtain

$$
d\left(x_{n}, x_{n+1}\right) \leq z_{n}(T)<\varepsilon e^{-\left(a+(n-1) a_{1}\right)} .
$$

In particular, $\left\{x_{n}\right\}$ is a Cauchy sequence and hence the segments $o x_{k}^{0}$ converge to a ray $\gamma$ as $k \rightarrow \infty$. Furthermore, for the point $x=\lim _{n} x_{n} \in \gamma$ we have $d(o, x)=T$ and

$$
d\left(x, x_{1}\right) \leq \sum_{n \geq 1} z_{n}(T)<\varepsilon-\varepsilon_{0},
$$

thus $d(x, \Omega) \geq \varepsilon_{0}$. Therefore, $d(\gamma, \Omega) \geq \varepsilon_{0}$. This completes the proof of Theorem 4.1 and Theorem 1.5 , respectively.

\section{Proof of Theorem 1.6}

We will first construct a singular metric $g$ with the required properties. The metric $g$ has the constant curvature -1 on the complement $M_{g} \backslash \Omega_{a}$, and the tangent cone $T_{\omega} M_{g}$ at each singular point $\omega \in \Omega_{a}$ is the Euclidean cone over the circle of length $3 \pi$. Second, we construct a $C^{\infty}$-smooth metric $g$ possessing the required properties by smoothing the 
singular metric. Of course, the Gaussian curvature of the smooth $g$ is not bounded from below.

We start with describing a standard block $\Omega \subset \mathbb{R}^{2}$ which we use to construct $\Omega_{a}$. In the following, we use the polar coordinates $(t, \phi) \in[0, \infty) \times[0,2 \pi)$ on $\mathbb{R}^{2}$ around the origin $o$.

Let $g_{0}$ be the metric of constant curvature -1 given by $d s_{0}^{2}=d t^{2}+(\sinh t)^{2} d \phi^{2}$, $M_{0}=\left(\mathbb{R}^{2}, g_{0}\right)$.

5.1. The construction of the standard block $\Omega$. We fix an integer $p \geq 3$ and define $\Omega=\cup_{n \geq 1} \Sigma_{n}$, where each $\Sigma_{n}$ lies in

$$
[n, n+1-1 / p] \times[0, \pi] .
$$

The set $\Sigma_{n}$ consists of $p^{n-1}$ subblocks $\Sigma_{n}^{i}, i=1, \ldots, p^{n-1}$, and the subblock $\Sigma_{n}^{i}$ is obtained by the counterclockwise rotation of $\Sigma_{n}^{1}$ around $o$ by the angle $\pi(i-1) / p^{n-1}$. It remains to describe the block $\Sigma_{n}^{1}$. It consists of $p$ points $\omega_{n}^{1}, \ldots, \omega_{n}^{p}$, where

$$
\omega_{n}^{i}=\left(n+(i-1) / p, \pi(i-1) / p^{n}\right) .
$$

We put $\Omega^{\prime}=\Omega \cup \sigma(\Omega)$, where $\sigma: \mathbb{R}^{2} \rightarrow \mathbb{R}^{2}$ is the reflection at $o$, and consider $\Omega, \Omega^{\prime}$ as subsets of $M_{0}$.

LEMMA 5.1. Every geodesic ray $\gamma:[0, \infty) \rightarrow M_{0}$ passes arbitrarily close to points of $\Omega^{\prime}$.

Proof. One can assume that $\gamma$ lies in the upper half-plane $\mathbb{H}_{+}^{2}=[0, \infty) \times[0, \pi]$. Let $\bar{\gamma}:[0, \infty) \rightarrow \mathbb{H}_{+}^{2}$ be the ray asymptotic to $\gamma$ with $\bar{\gamma}(0)=o$. By reparametrizing $\gamma$ we can assume that $d(\gamma(t), \bar{\gamma}(t)) \rightarrow 0$ for $t \rightarrow \infty$.

The radial projection $\bar{\Sigma}_{n}$ of $\Sigma_{n}$ to the half-circle $S_{n+1}=\{n+1\} \times[0, \pi]$ consists of $p^{n}$ points

$$
\bar{\omega}_{n}^{i}=\left(n+1, \pi(i-1) / p^{n}\right), \quad i=1, \ldots, p^{n},
$$

which subdivide $S_{n+1}$ into $p^{n}$ equal arcs. Thus

$$
d\left(\bar{\gamma}_{[[n, n+1]}, \Sigma_{n}\right) \leq d\left(\bar{\gamma}_{\mid[n, n+1]}, \bar{\Sigma}_{n}\right) \leq \frac{\pi}{p^{n}} \sinh (n+1) \rightarrow 0
$$

as $n \rightarrow \infty$, since $p \geq 3>e$. Because the Hausdorff distance $\operatorname{Hd}\left(\gamma_{\mid[n, n+1]}, \bar{\gamma}_{\mid[n, n+1]}\right) \rightarrow 0$ for $n \rightarrow \infty$ we conclude that $d\left(\gamma, \Sigma_{n}\right) \rightarrow 0$.

5.2. The construction of a singular metric $g$. We take $a=1 / p$. The set $\Omega^{\prime} \subset M_{0}$ constructed in $\S 5.1$ satisfies $d\left(o, \Omega^{\prime}\right)=1 \geq a$, and any $\omega, \omega^{\prime} \in \Omega^{\prime}$ with distinct distances from $o$ are separated by a distance $\geq a$. However, $\Omega^{\prime}$ is not an $a$-separated set. Points $\omega \in \Sigma_{n}^{i}, \omega^{\prime} \in \Sigma_{n}^{i+1}$ with $d(o, \omega)=d\left(o, \omega^{\prime}\right)$ are arbitrarily close to each other for $n$ sufficiently large.

To remedy this, we make a slit $s_{\omega}$ along the ray $\left[t_{\omega}, \infty\right) \times\left\{\phi_{\omega}\right\}$ for each $\omega \in \Omega^{\prime}$, $\omega=\left(t_{\omega}, \phi_{\omega}\right)$, and glue in it a copy of the upper half-plane $\mathbb{H}_{+}^{2}$. For that, we identify the right-hand side $s_{\omega}^{+}$of $s_{\omega}$ with the ray $[0, \infty) \times\{0\} \subset \partial \mathbb{H}_{+}^{2}$ and the left-hand side $s_{\omega}^{-}$of $s_{\omega}$ 
with $[0, \infty) \times\{\pi\} \subset \partial \mathbb{H}_{+}^{2}$. We perform this starting with the lowest level points of $\Omega^{\prime}$, for which $t_{\omega}=1$, and assume every time that if $\omega^{\prime} \in \Omega^{\prime}$ lies on the slit ray $\left[t_{\omega}, \infty\right) \times\left\{\phi_{\omega}\right\}$, then $\omega^{\prime}$ belongs to the left-hand side $s_{\omega}^{-}$of $s_{\omega}$.

We call this construction the blow-up at $\Omega^{\prime}$. It defines a complete singular metric $g_{1}$ on $\mathbb{R}^{2}$ of curvature $\leq-1$. Its singular set $\Omega_{1}$ can be identified with $\Omega^{\prime}$ via the obvious projection $M_{1}=\left(\mathbb{R}^{2}, g_{1}\right) \rightarrow M_{0}$. The metric $g_{1}$ has the constant curvature -1 on the complement $M_{1} \backslash \Omega_{1}$, and the space of directions at any $\omega \in \Omega_{1}$ is the circle of length $3 \pi$.

The blow-up at $\Omega^{\prime}$ separates each pair of blocks $\Sigma_{n}^{i}, \Sigma_{n}^{i+1}, n \geq 1$, and thus $\Omega_{1}$ is $a$-separated. It follows from the construction and Lemma 5.1 that each geodesic ray $\gamma:[0, \infty) \rightarrow M_{1}, \gamma(0)=o$, passes arbitrarily close to points of $\Omega_{1}$. Hence, every ray in $X$ passes arbitrarily close to points of $\Omega_{1}$. This completes the proof of Theorem 1.6 for the case of a singular metric $g=g_{1}$.

5.3. The construction of a smooth metric $g$. We construct a smooth metric $g$ from Theorem 1.6 by smoothing a singular metric of the type described above in small neighborhoods of singular points. However, to use the metric $g_{1}$ from $\$ 5.2$ would not be appropriate, because for any smoothed metric $g_{1}^{\prime}$ there exist geodesic rays starting at $o$, missing $\Omega_{1}$ and with tails lying inside of glued sectors $\mathbb{H}_{+}^{2}$, i.e. far away from $\Omega_{1}$. Therefore, we need to modify $g_{1}$. To this end, we recall that the upper half-plane $\mathbb{H}_{+}^{2}$ carries an additional piece of information, namely, the standard block $\Omega$.

5.3.1. Construction of a singular metric $g_{\infty}$. We start with the metric $g_{1}$ (see \$5.2). The space $M_{1}$ contains a copy $H_{\omega}$ of $\mathbb{H}_{+}^{2}$ glued into the slit $s_{\omega}$ for each $\omega \in \Omega_{1}$. By the construction, $H_{\omega}$ contains only two points of $\Omega_{1}$ both lying on the left-hand side of $\partial H_{\omega}$ : $\omega$ and $\omega^{\prime}$ with $d\left(\omega^{\prime}, \omega\right)=1$. Using the isometry $\mathbb{H}_{+}^{2} \rightarrow H_{\omega}$, we obtain a copy $\Omega_{\omega}$ of the standard block $\Omega$ in $H_{\omega}$. It follows that $\Omega_{\omega} \cap \Omega_{1}=\emptyset$ for each $\omega \in \Omega_{1}$.

Now we define

$$
\Omega_{2}^{\prime}=\Omega_{1} \cup \bigcup_{\omega \in \Omega_{1}} \Omega_{\omega}
$$

and make the blow-up at $\Omega_{2}^{\prime} \backslash \Omega_{1}$. This gives a singular metric $g_{2}$ on $\mathbb{R}^{2}$, whose singular set $\Omega_{2}$ can be identified with $\Omega_{2}^{\prime}$ via the projection $M_{2}=\left(\mathbb{R}^{2}, g_{2}\right) \rightarrow M_{1}$. Again, $\Omega_{2}$ is $a$-separated in $M_{2}$. Proceeding by induction, we obtain sequences of sets

$$
\Omega_{n}^{\prime}=\Omega_{n-1} \cup \bigcup_{\omega \in \Omega_{n-1}} \Omega_{\omega},
$$

singular metrics $g_{n}$ on $\mathbb{R}^{2}$ with singular sets $\Omega_{n} \simeq \Omega_{n}^{\prime}$, and each $\Omega_{n}$ is $a$-separated in $M_{n}=\left(\mathbb{R}^{2}, g_{n}\right)$. The metric $g_{n}$ has the constant curvature -1 on the complement $M_{n} \backslash \Omega_{n}$, and the space of directions at any $\omega \in \Omega_{n}$ is the circle of length $3 \pi$.

LEMMA 5.2. The sequence $\left\{g_{n}\right\}$ converges uniformly on compact subsets of $\mathbb{R}^{2}$ to a complete singular metric $g_{\infty}$ of curvature $\leq-1$. The singular set $\Omega_{\infty}$ of $g_{\infty}$ is aseparated, $d\left(o, \Omega_{\infty}\right)=1, g_{\infty}$ has the constant curvature -1 on the complement $M_{\infty} \backslash \Omega_{\infty}$, where $M_{\infty}=\left(\mathbb{R}^{2}, g_{\infty}\right)$, and the space of directions at any $\omega \in \Omega_{\infty}$ is the circle of length $3 \pi$. 
Proof. This follows from the fact that

$$
d\left(o, \Omega_{n+1}^{\prime} \backslash \Omega_{n}\right)=n+1
$$

for each $n \geq 1$, and hence the blow-up at $\Omega_{n+1}^{\prime} \backslash \Omega_{n}$ changes nothing in the ball of radius $n$ centered at $o$.

5.3.2. Smoothing the metric $g_{\infty}$. Let $\varepsilon: \Omega_{\infty} \rightarrow \mathbb{R}_{+}$be a positive function such that $\varepsilon(\omega)$ depends only on the distance $d(o, \omega)$ in $M_{\infty}$ and $\varepsilon(\omega) \rightarrow 0$ as $d(o, \omega) \rightarrow \infty$. We assume, also, that $\varepsilon(\omega)<a / 2$ for all $\omega \in \Omega_{\infty}$. Smoothing the metric $g_{\infty}$ in a neighborhood $U_{\omega}$ of radius $<\varepsilon(\omega)$ for each $\omega \in \Omega_{\infty}$, we obtain a $C^{\infty}$-smooth metric $g_{\varepsilon}$ of curvature $\leq-1$. We denote $M_{\varepsilon}=\left(\mathbb{R}^{2}, g_{\varepsilon}\right)$.

There is a 1-Lipschitz homeomorphism pro $_{\infty}: M_{\varepsilon} \rightarrow M_{\infty}$, which is an isometry on the complement

$$
M_{\infty} \backslash \bigcup_{\omega \in \Omega_{\infty}} U_{\omega}
$$

because the smoothing is local and does not decrease distances. Using it, we identify $\Omega_{\infty}$ with $\Omega_{a} \subset M_{\varepsilon}$. Thus $\Omega_{a}$ is $a$-separated and $d\left(o, \Omega_{a}\right) \geq a$.

Proposition 5.1. Every geodesic ray $\gamma:[0, \infty) \rightarrow M_{\varepsilon}$ passes arbitrarily close to points of $\Omega_{a}$.

Proof. Suppose that the assertion is not true, and there is a $\delta>0$ such that $d\left(\gamma, \Omega_{a}\right) \geq 2 \delta$ for some ray $\gamma$ in $M_{\varepsilon}$.

By construction (see §5.3.1), the singular set $\Omega_{\infty} \subset M_{\infty}$ is the union of $\Omega_{n}, n \geq 1$, the singular sets of metrics $g_{n}$, and $\Omega_{n} \subset \Omega_{n+1}$ for each $n \geq 1$. We grade $\Omega_{\infty}$ by the rule $\operatorname{deg}(\omega)=\inf \left\{n \mid \omega \in \Omega_{n}\right\}$ for $\omega \in \Omega_{\infty}$. Using the obvious projection $\operatorname{pr}_{n}: M_{\infty} \rightarrow M_{n}$, we denote

$$
H_{\omega}^{\infty}=\operatorname{pr}_{n}^{-1}\left(H_{\omega}\right)
$$

for $\omega \in \Omega_{\infty}$ of degree $n$.

By the assumption, the curve $\gamma_{\infty}=\operatorname{pro}_{\infty} \circ \gamma$ in $M_{\infty}$ has a geodesic tail $\gamma_{\delta}$, whose $\delta$-neighborhood misses the singular set $\Omega_{\infty}$, because $\varepsilon(\omega) \rightarrow 0$ as $d(o, \omega) \rightarrow \infty$. Every $H_{\omega}^{\infty} \subset M_{\infty}$ is convex and its boundary is geodesic. Hence, after entering an $H_{\omega}^{\infty}$ the geodesic $\gamma_{\delta}$ never leaves it. Let

$$
n_{\gamma}=\sup \left\{\operatorname{deg}(\omega) \mid \gamma_{\delta} \cap H_{\omega}^{\infty} \neq \emptyset\right\}
$$

First, we consider the case $n=n_{\gamma}<\infty$. Without loss of generality, one can assume that $\gamma_{\delta} \subset H_{\omega}^{\infty}$ with $\operatorname{deg}(\omega)=n$. Projecting $\gamma_{\delta}$ into $M_{n}$ via $\operatorname{pr}_{n}$ and using the same notation $\gamma_{\delta}$ for the image, we obtain that $\gamma_{\delta} \subset H_{\omega}$ passes at a distance at least $\delta$ from $\Omega_{\omega}$ (see §5.3.1). This contradicts Lemma 5.1.

Assume, second, that $n_{\gamma}=\infty$. Hence, $\gamma_{\delta}$ enters $H_{\omega}^{\infty}$ for arbitrarily large $\operatorname{deg}(\omega)$. Let $\left\{H_{k}=H_{\omega_{k}}^{\infty}\right\}$ be the corresponding sequence of consecutive sectors, $\alpha_{k}$ the angle formed by $\gamma_{\delta}$ with the corresponding boundary ray $s_{\omega_{k}}^{ \pm}$of $H_{k}$ at the entering point $x_{k}$ into $H_{k}$ (recall that $\gamma_{\delta}$ misses the $\omega_{k}$ and, hence, $x_{k} \neq \omega_{k}$ ). Projecting the picture into the hyperbolic half-plane $H_{\omega_{k}}$ via $\operatorname{pr}_{n}, n=\operatorname{deg}\left(\omega_{k}\right)$, we consider the triangle $\triangle_{k}:=x_{k} x_{k+1} \omega_{k} \subset H_{\omega_{k}}$. 
Note that $\omega_{k+1}$ is a point on the segment $\omega_{k} x_{k+1}$. Let $a_{k}$ be the area of the triangle $\triangle_{k}$ and $\sigma_{k}:=L_{\omega_{k}}\left(x_{k}, x_{k+1}\right)=L_{\omega_{k}}\left(x_{k}, \omega_{k+1}\right)$. By the theorem of Gauss-Bonnet we have

$$
\alpha_{k+1}=\alpha_{k}-\left(a_{k}+\sigma_{k}\right)
$$

and hence

$$
a_{k}, \sigma_{k} \longrightarrow 0 \text { for } k \longrightarrow \infty \text {. }
$$

First observe that the interior of $\triangle_{k}$ does not contain any $\omega \in \Omega_{\omega_{k}}$ since the segment $x_{k} x_{k+1}$ cannot intersect a slit of $H_{\omega_{k}}$. From the construction of the set $\Omega_{\omega_{k}}$ there exists a point $\omega_{k}^{\prime} \in \Omega_{\omega_{k}}$ contained in the angular sector determined by the geodesic segments $\omega_{k} x_{k}$ and $\omega_{k} x_{k+1}$ with

$$
d\left(\omega_{k}^{\prime}, \omega_{k+1}\right) \leq 1
$$

Since $\omega_{k}^{\prime}$ is not in the interior of $\triangle_{k}$, the segments $x_{k} x_{k+1}$ and $\omega_{k}^{\prime} \omega_{k+1}$ intersect in a point $p_{k}$. As $\sigma_{k} \rightarrow 0$ by (i) the construction of the set $\Omega_{\omega_{k}}$ implies that

$$
d\left(\omega_{k}, \omega_{k+1}\right) \longrightarrow \infty .
$$

By the choice of $\delta$ we have $d\left(\omega_{k+1}, x_{k} x_{k+1}\right) \geq \delta$. From this together with $\sigma_{k} \rightarrow 0$ and $d\left(\omega_{k}, \omega_{k+1}\right) \rightarrow \infty$ we infer that

$$
d\left(\omega_{k}, x_{k}\right) \longrightarrow \infty
$$

Because of $d\left(\omega_{k+1}, x_{k+1}\right) \rightarrow \infty$ by (iv) we obtain by using (ii) that

$$
d\left(x_{k+1}, p_{k}\right) \longrightarrow \infty \text {. }
$$

Since $a_{k}=\operatorname{area}\left(\triangle_{k}\right) \rightarrow 0$ by (i), the area of the triangle $p_{k} x_{k+1} \omega_{k+1}$ goes a fortiori to zero if $k$ tends to infinity. Now we use $d\left(x_{k+1}, p_{k}\right) \rightarrow \infty$ (by (v)) and $d\left(\omega_{k+1}, p_{k}\right) \geq \delta$ by the choice of $\delta$ together with area $\left(p_{k} x_{k+1} \omega_{k+1}\right) \rightarrow 0$ to conclude that the triangle $p_{k} x_{k+1} \omega_{k+1}$ is 'almost degenerated', i.e. for a subsequence $\left(k_{j}\right)$ of $(k)$ either $\tau_{k_{j}}:=$ $L_{p_{k_{j}}}\left(x_{k_{j}+1}, \omega_{k_{j}+1}\right) \rightarrow 0$ or $\tau_{k_{j}} \rightarrow \pi$ for $j \rightarrow \infty$. If $\tau_{k_{j}} \rightarrow 0$ then $d\left(p_{k_{j}}, x_{k_{j}+1}\right) \rightarrow \infty$ (by (v)) and $d\left(p_{k_{j}}, \omega_{k_{j}+1}\right) \leq 1$ (by (ii)) imply that $d\left(\omega_{k_{j}+1}, p_{k_{j}} x_{k_{j}+1}\right) \rightarrow 0$ for $j \rightarrow \infty$. If $\tau_{k_{j}} \rightarrow \pi$ we conclude analogously that $d\left(\omega_{k_{j}}^{\prime}, p_{k_{j}} x_{k_{j}+1}\right) \rightarrow 0$ for $j \rightarrow \infty$. Both alternatives contradict the property $d\left(\gamma_{\delta}, \Omega_{\infty}\right) \geq \delta$.

This completes the proof of Theorem 1.6 for the case of a smooth metric $g=g_{\varepsilon}$.

Acknowledgements. Parts of the results were obtained in the thesis of the third author $[\mathbf{W}]$. We thank C. Hummel for inspiring discussions. The first author is grateful to FIM ETH Zürich for hospitality and excellent working conditions. We are very grateful to the referee for the detailed comments and suggestions. SB was Supported by RFFI Grants 96-01-00674, 96-15-00675 and CRDF Grant RM1-169.

\section{REFERENCES}

[Ba] W. Ballmann. Lectures on Spaces of Nonpositive Curvature (DMV-Seminar, 25). Birkhäuser, 1995.

[BP] K. Burns and M. Pollicott. Self-intersections of geodesics and projecting flow invariant sets. Preprint, 1994.

[W] M. Walz. Invariant subsets of the geodesic flow on negatively curved manifolds. Thesis, University of Zürich, 1998. 